\title{
Comparative Analysis of Quad-tree Partitioned Iterated Function Systems for Medical Images
}

\author{
S.Padmashree \\ Associate Professor \\ Dept. of ECE \\ GSSSIETW, Mysore
}

\author{
Rohini Nagapadma \\ Professor \\ Dept. of ECE \\ The National Institute of Engg, Mysore
}

\begin{abstract}
As Image size need to be reduced for the purpose of data storage and transmission for better utilization of the Bandwidth, Images are compressed using lossy and Lossless compression schemes. In this paper Fractal Image Compression, a lossy compression technique is being implemented on medical Images .Fractal Encoding involves partitioning the images into Range Blocks and Domain Blocks and each Range Block is mapped onto the Domain Blocks by using contractive transforms called the Affine Transforms. The Fractal encoding technique takes a longer encoding time and less decoding time. In the present paper Fractal Image Compression using quad-tree Partitioning technique is being implemented. on Medical Images like CT Of Bone and MR Images of Brain The Performance measures like Compression Ratio (CR), Peak Signal To Noise Ratio (PSNR), Mean Square Error (MSE), Encoding time and Decoding Time are determined for the Range Blocks of Sizes 2x2 and 4x4 respectively with different Threshold Values. Mat lab simulated results for these Performance Measures shows that for larger Range Block size, the PSNR Value decreases and CR increases for different threshold Values.
\end{abstract}

\section{General Terms}

Study of Fractal Image Compression on Medical Images.

\section{Keywords}

Fractal Theory; Iterated Function systems; quad-tree; Range blocks.

\section{INTRODUCTION}

Benoit. B.Mandelbrot, a mathematician first developed Fractal theory in Mathematical geometry which was further developed by M Barnsley who introduced the fundamental principle of fractal image compression in the year 1988 [1]. Fractal image compression is also called fractal image encoding as the compressed image is represented by contractive transforms and mathematical functions which are required for reconstruction of the image [2]. Contractive transforms ensures that the distance between any two points on the transformed image will be less than the distance between the same points on the original image. These transforms are composed by the union of number of affine mappings on the entire image known as the Iterated Function Systems (IFS) [2-4]. The fractal image compression based on IFS was complicated and was still not practical. Arnaud Jacquin settled the problem with the partition of IFS in 1990. .Jacquin partitioned the image into sub images called the Range Blocks and partitioned iterated function systems (PIFS) were applied on the sub images rather than the whole image. Temporary images used to form the range blocks are called the domain blocks. The whole process of fractal image encoding involves Partitioning the image to form the Range blocks and domain blocks [4], detection of domain blocks [5-
6] and Proper application of affine transforms. Further the choice of domain blocks depends on the type of partition scheme used. The domain pool in fractal encoding is similar to the codebook in vector quantization (VQ) which is referred to as virtual codebook or domain codebook. The set of transformations selected is applied on each Range block to map on to the domain blocks. As the encoding time is larger than the decoding time fractal coding is asymmetric in nature.

\section{FRACTAL IMAGE COMPRESSION}

Fractal Image Compression is based on fractal theory of self similar affine transforms [7].

\subsection{Self similar and Self Affine transforms}

In this section, theory involved in Fractal Image Compression is being discussed. It is basically based on fractal theory of self-affine transformations and self-similar transformations [7]. A self-affine transformation w: defined by $R^{n} \rightarrow R^{n}$ transformation is of the form $w(x)=T(x)+b$, where $T$ is a linear transformation on $R^{n}$ and $b \in R^{n}$ is a vector. If there exists a mapping $\mathrm{w}$ of the form $\mathrm{D} \rightarrow \mathrm{D}$ and $\mathrm{D}$ is a subset of ,$R^{n}$ then $\mathrm{D}$ is a contraction of w such that $d(w(x), w(y)) \leq$ $c d(x, y)$ for $x, y \in D$ and for a metric $d$ on $R^{n}$. The real number $c$ is called the contractivity of $w$. If $d(w(x), w(y))=c d(x, y)$ then $w$ is called a similarity. A family of $\left\{w_{1}, w_{2} \cdots w_{m}\right\}$ of contractions is known as Local Iterated function scheme (LIFS). Once the LIFS is formed, the encoding of the image can be obtained so that, the attractor is similar to the original image. Barnsley's collage theorem tells how well the attractor can be an approximated to the original image. This theorem says that if a transformation is contractive, then when applied repeatedly starting with any initial point, then the point converges to a unique fixed point. This simple looking theorem tells us how the collection of transformations defines an image.

\subsection{Fractal Encoding Algorithm}

- Input Image is loaded into the buffer

- The Image is partitioned into non overlapping square blocks called the Range blocks

- The Initial size of the domain blocks is chosen to be twice the size of the range block

- The domain blocks is down sampled to the size of range blocks and the eight possible affine transformations is computed for each Range block

- The domain block chosen, shall resemble the range block with respect to some metric and accordingly encoding parameters are computed .The decoding time generally depends on the number of Iterations and here it takes only few iterations ranging from $4-8$ to reach the fixed point

The reconstruction process of the original image consists of the applications of the transformations described in the 
fractal code book iteratively to some initial image until the encoded image is retrieved back

\section{QUADTREE PARTITIONING}

The most popular partitioning mechanism in fractal Image compression (FIC) is obtained by partitioning the image in a tree structure called quad-tree partitioning method. A quadtree partitioning [8-9] is a representation of an image as a tree in which each node corresponding to a square portion of the image contains four sub-nodes corresponding to the four quadrants of the square, the root of the tree being the initial image as in figure 1 shown below.

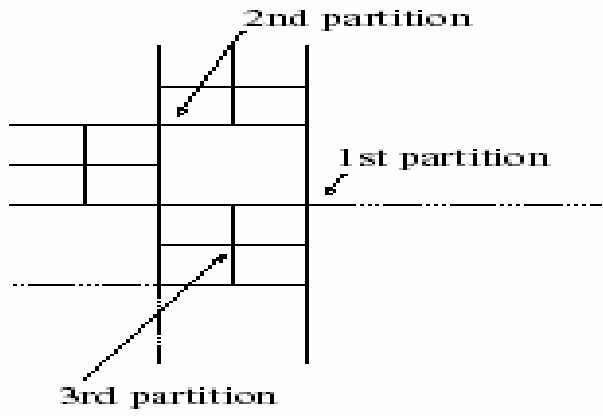

Figure 1: Quadtree partitioning

\subsection{Implementation of the Algorithm}

- The Input image to be compressed is read.

- The original image is partitioned into Range blocks according to Quad-tree partitioning method which divides a square image into four equal-sized square blocks, and then each block is tested to see if it meets some criteria of homogeneity. If the block meets the criteria, it is not divided any further. If it does not meet the criteria, it is subdivided again into four blocks, and the test criteria are applied to those blocks.

- This process is repeated iteratively until each block meets the criteria. The result may have Range blocks of several different sizes. The criteria met in the present quad-tree method is that, the partitioning method will iteratively divide until it reaches the minimum block dimension of blocks. In this paper minimum Range block sizes of $2 \times 2$ and 4 X4 has been chosen for the implementation.

- Affine transformation is applied to each of the domain block and is compared with each of the Range block and a closest approximation of the domain block with that of the Range block is found based on the Root mean squared error calculated between the Range Blocks and the transformed domain Blocks.

- The transformed domain block which is found to be the best approximation for the current range block is assigned to that range block and this forms the fractal code book.

- The reconstruction process of the original image consists on the applications of the transformations described in the fractal code book iteratively to some initial image until the encoded image is retrieved back. PSNR, CR [10], Encoding time, Decoding time and MSE are calculated for the further analysis of the image

\section{RESULTS AND DISCUSSION}

Forty images out of which twenty images of Magnetic Resonance Images [11] of brain (T1 and T2 weighted) and twenty images of CT images [11] of bones of size 256x256 of
8 bit gray scale were collected from JSS Hospital and Vikram Hospital Mysore. Fractal image compression using quad-tree partitioning was applied on the images with the threshold varying from 0.1 to 0.4 .The minimum Range Block sizes were taken to be 2 and 4. Compression Ratio (CR), Peak Signal to Noise Ratio (PSNR), Mean Square Error (MSE), Encoding and decoding time were computed for the images and a comparison of the PSNR, encoding time and decoding time for a minimum Range block size of $2 \mathrm{X} 2$ and $4 \times 4$ were carried out.

Table1 below shows the statistical comparison of these performance measures From the Table 1 it is seen that when the Range Block size is increased from $2 \mathrm{X} 2$ to $4 \mathrm{X} 4$, the Compression Ratio gets increased from 5.3 to 17.9 for MR Image of Brain and for CT image of Bone. Also the PSNR gets decreased to 29.8 from $32.8 \mathrm{db}$.

TABLE 1: Comparison of PSNR, CR MSE of CT and MR Images for Different Thresholds and Range Blocks.

\begin{tabular}{|c|c|c|c|c|c|c|c|}
\hline \multirow[t]{2}{*}{$\begin{array}{l}\text { Threshol } \\
\text { d }\end{array}$} & \multirow{2}{*}{$\begin{array}{l}\text { Rang } \\
\text { e } \\
\text { Block } \\
\text { s }\end{array}$} & \multicolumn{2}{|l|}{ CR } & \multicolumn{2}{|c|}{$\begin{array}{l}\text { PSNR(dB } \\
\text { ) }\end{array}$} & \multicolumn{2}{|c|}{ MSE (dB) } \\
\hline & & $\begin{array}{l}\mathbf{M} \\
\mathbf{R}\end{array}$ & $\begin{array}{c}\mathrm{C} \\
\mathrm{T}\end{array}$ & $\begin{array}{l}\mathbf{M} \\
\mathbf{R}\end{array}$ & CT & MR & CT \\
\hline 0.1 & $2 \times 2$ & 5.3 & 5.4 & $\begin{array}{l}32 . \\
8\end{array}$ & $\begin{array}{l}32 . \\
1\end{array}$ & $\begin{array}{l}136 . \\
5\end{array}$ & $\begin{array}{l}162 . \\
4\end{array}$ \\
\hline 0.2 & $2 \times 2$ & 6.5 & 7.9 & $\begin{array}{l}32 . \\
6\end{array}$ & $\begin{array}{l}28 . \\
3\end{array}$ & $\begin{array}{l}144 . \\
6\end{array}$ & $\begin{array}{l}169 . \\
8\end{array}$ \\
\hline 0.3 & $2 \times 2$ & 7.6 & 7.9 & $\begin{array}{l}31 . \\
8\end{array}$ & $\begin{array}{l}31 . \\
6\end{array}$ & $\begin{array}{l}172 . \\
6\end{array}$ & $\begin{array}{l}180 . \\
2\end{array}$ \\
\hline 0.1 & $4 \times 4$ & $\begin{array}{l}17 . \\
9\end{array}$ & $\begin{array}{l}17 . \\
9\end{array}$ & $\begin{array}{l}29 . \\
8\end{array}$ & $\begin{array}{l}28 . \\
3\end{array}$ & $\begin{array}{l}274 . \\
4\end{array}$ & $\begin{array}{l}386 . \\
2\end{array}$ \\
\hline 0.2 & $4 \times 4$ & $\begin{array}{l}20 . \\
4\end{array}$ & $\begin{array}{l}21 . \\
6\end{array}$ & $\begin{array}{l}29 . \\
8\end{array}$ & $\begin{array}{l}28 . \\
3\end{array}$ & $\begin{array}{l}277 . \\
1\end{array}$ & $\begin{array}{l}389 . \\
3\end{array}$ \\
\hline 0.3 & $4 \times 4$ & $\begin{array}{l}23 . \\
7\end{array}$ & $\begin{array}{l}23 . \\
3\end{array}$ & $\begin{array}{l}29 . \\
6\end{array}$ & $\begin{array}{l}28 . \\
2\end{array}$ & $\begin{array}{l}290 . \\
2\end{array}$ & $\begin{array}{l}393 . \\
5\end{array}$ \\
\hline
\end{tabular}

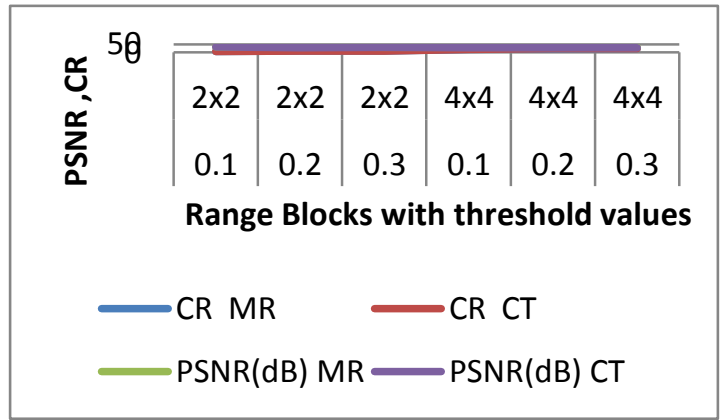

Figure 2: variation of PSNR and CR with Range Blocks $2 \times 2$ and $4 \times 4$ for different threshold values ranging from 0.1 to 0.4

It is observed from the Graph of figure. 2 that an increase in the Range Block size results in decrease in the PSNR value and increase in $\mathrm{CR}$ value. This effects the quality of the medical Images as High CR may result in loss of some useful information. Hence increase in range block size is not advisable for medical images and from this perspective a minimum Range Block size of $2 \mathrm{X} 2$ is preferred for applying 
quad tree partitioning technique on medical images like MR Image of Brain and CT.

IMAGE FOR DIFFERENT RANGE BLOCKS

Original image quad-tree reconstructed image
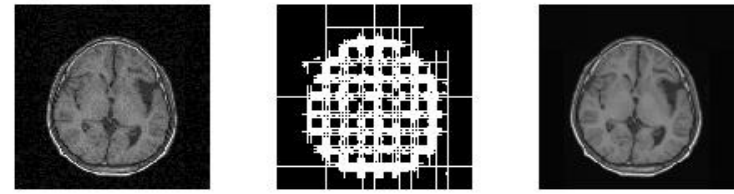

Figure 3a): Quad-tree FIC on MR Image for Range Block 2X2, Threshold 0.1

Original image

quad-tree

reconstructed image
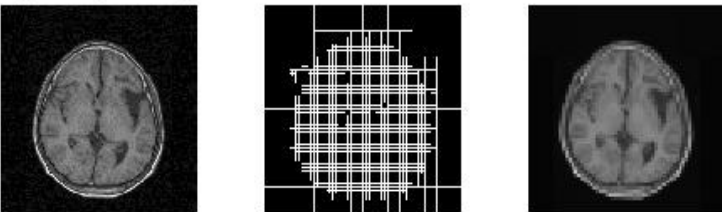

Figure 3b): Quad-tree FIC on MR Image for Range Block 4X4, Threshold 0.1

Original Image

quad-tree reconstructed image
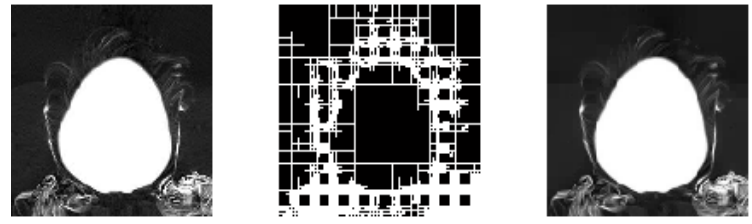

Figure 3c): Quad-tree FIC on CT Image for Range Block 2X2, Threshold 0.1

Original quad-tree reconstructed image
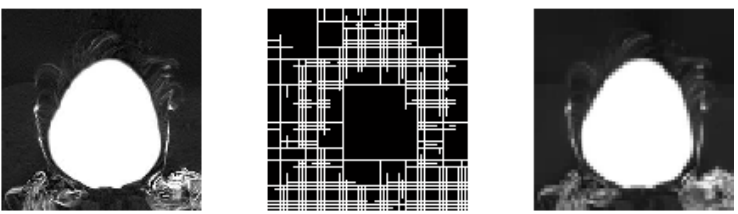

Figure 3d): Quad-tree FIC on CT Image for Range Block 4X4, Threshold 0.1

Original quad-tree reconstructed image
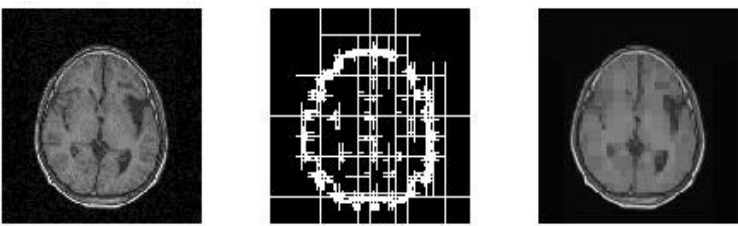

Figure 3e): Quad-tree FIC on MR Image for Range Block 2X2, Threshold 0.3

The figures 3a-3e shows the quad-tree partitioned image for medical images and the reconstructed image. It is seen from the results that quad tree partitioning technique in fractal encoding, has preserved the information in both the medical images. Also the technique has brought in compression of the image when the range block size is $2 \times 2$. However for range block size of $4 \times 4$, as the compression ratio and signal to noise ratio are higher, it is advisable to consider the range block size to be $2 \times 2$, to better suit for medical images especially MR Image of Brain.

The above algorithms are simulated using MATLAB 7.12 and the programs are executed on personal computer with processing speed of $2.2 \mathrm{GHZ}$. The Processor is core 2 duo and has 2GB RAM .The time taken for fractal encoding is $6.7 \mathrm{Sec}$ and time taken for decoding is $0.5905 \mathrm{sec}$

\section{CONCLUSIONS}

From the Simulated results it is observed that the PSNR value decreases with increase in threshold values for MR Images and CT images. Further, the CR and threshold are directly proportional, to each other. When the threshold value is high the quality of the images gets degraded as the compression ratio increases and some useful information is lost. Hence an optimum threshold [12] should be chosen to maintain the quality of the image. In this paper a threshold of 0.1 and 0.2 provides good quality compressed images. The average encoding time and decoding time for MR images using quadtree FIC are $6.7 \mathrm{sec}$ and $0.59 \mathrm{sec}$. respectively.

\section{REFERENCES}

[1] M. Barnsley, Fractals Everywhere. New York: Academic,(1988).

[2] A.E. Jacquin, "Image coding based on a fractal theory of iterated contractive image transformation", IEEE Trans. On Image Processing, 1(1): (1992)

[3] Arnaud E. Jacquin, (1993) "Fractal image coding", Proceedings of IEEE VOL.81, pp. 1451-1465

[4] Y. Fisher, Fractal Image Compression: Theory and Application. New York: Springer-Verlag, (1994).

[5] R. Distasi, M. Nappi and D. Riccio, "A range/domain approximation error- based approach for fractal image compression", IEEE Trans. Image processing, vol. 15, no. 1, pp. 89-97, Jan. 2006.

[6] Vijayshri Chaurasia et al. / International Journal of Engineering Science and Technology Vol.2 (2), 2010, 104-108

[7] Y. Chakrapani, K. Rajan. "Implementation of fractal image compression employing artificial neural networks ISSN 1 746-7233, England, UK World Journal of Modeling and Simulation Vol. 4 (2008) No. 4, pp. $287-$ 295

[8] Dr. Fakhiraldeen H. Ali Quad-tree Fractal Image Compression University of Mosul

[9] M.S.Soyjaudah and I.Jahmeerbacus "Fractal image compression using quad-tree partitioning" International Journal of Electrical Engineering Education 39/1

[10] Pamela,,Gray,R.M.olshenA.(1994b)“Evaluating Quality of Compressed Medical Images: SNR, Subjective Rating and Diagnostic Accuracy', Proc. of the IEEE, Vol. 82, pp. 920-931.

[11] S. Bhavani et. al. / (IJCSE) International Journal on Computer Science and Engineering Vol. 02, No. 05, 2010, 1429-1434 A Survey On Coding Algorithms In Medical Image compression

[12] Sumathi Poobaland G. Ravindran, "Arriving at an OptimumValue of Tolerance Factor for Compressing Medical Images," world Academy of Science, Engineering and Technology, vol. 24, pp. 169-173, 2006. 